\title{
COMPOSICIÓN FLORÍSTICA EN POTREROS DE LOS TUXTLAS, VERACRUZ, MÉXICO
}

\author{
Andrés Lira-Noriega, Sergio Guevara, JaVier Laborde y Graciela Sánchez-Ríos
}

Instituto de Ecología, A.C., Departamento de Ecología Funcional

km 2.5 Carretera antigua a Coatepec Núm. 351, Congregación El Haya

91070 Xalapa, Veracruz, México. aliranoriega@yahoo.com

\section{RESUMEN}

Se hizo un inventario de especies de plantas fanerógamas y pteridofitas en potreros en áreas desprovistas de árboles, en la zona del volcán San Martín Tuxtla, en Los Tuxtlas, Veracruz. Con el fin de tener la composición florística más completa posible, se realizaron muestreos durante la temporada seca y la de lluvia del año de 1992, en dos localidades: el ejido Balzapote y la colonia agrícola-ganadera La Palma. En cada localidad se seleccionaron cinco potreros dominados por especies de pastos nativas (conocidos como potreros de grama) y cinco en que prevalece la gramínea introducida Cynodon plectostachyus (llamados potreros de estrella). En cada potrero, dentro de una hectárea se ubicaron al azar 10 cuadros fijos de 2 x $2 \mathrm{~m}$ en los que se registró a toda planta presente y se estimó su cobertura, además de medir la altura de la vegetación dentro de cada cuadro. El resultado arrojó un total de 200 especies de angiospermas y 14 de pteridofitas, pertenecientes a 55 familias. Las familias con 10 especies o más fueron Asteraceae, Euphorbiaceae, Poaceae y Leguminosae (sensu lato). Las plantas con cobertura mayor de $10 \%$ del área muestreada fueron C. plectostachyus, Axonopus spp., Paspalum conjugatum (Poaceae), Hyptis atrorubens (Lamiaceae) y Mimosa pudica (Leguminosae: Mimosaceae). La mayoría de las especies encontradas en potreros fueron malezas y pastos; sin embargo, también se registraron plántulas de árboles y arbustos.

Palabras clave: deforestación, manejo agropecuario, potrero, riqueza específica, trópico húmedo.

\section{ABSTRACT}

An inventory of the angiosperm and fern plant species growing in areas deprived of trees in pastures of Los Tuxtlas mountain range is presented. In order to obtain a complete floristic list, sampling was carried out during both the dry and wet seasons of 1992, in pastures 
of two localities: Balzapote and La Palma. At each locality, five pastures dominated by native grass species (known locally as grama pastures) and five dominated by the introduced African star grass, Cynodon plectostachyus (known as star pastures), were selected. In each pasture ten $2 \times 2$ m sampling plots were randomly established within one hectare, recording the cover of every species present within each quadrat, in addition vegetation height was measured. A total of 200 angiosperm and 14 fern species, belonging to 55 families, was recorded. The families with 10 or more species were Asteraceae, Euphorbiaceae, Poaceae and Leguminosae (sensu lato). The species with cover greater than $10 \%$ of sampled area were C. plectostachyus, Axonopus spp., Paspalum conjugatum (Poaceae), Hyptis atrorubens (Lamiaceae), and Mimosa pudica (Leguminosae: Mimosaceae). Most species found in pastures were weeds and grasses; however, seedlings of tree and shrub species were also found. richness.

Key words: agricultural management, deforestation, humid tropics, pasture, species

\section{INTRODUCCIÓN}

En el trópico húmedo de México la superficie de potreros ha ido en aumento en relación directa con el incremento de la deforestación de la selva. En esta región se estima que la cobertura forestal ha sido reducida a menos de $20 \%$ de su extensión original debido a las actividades agrícolas (equivalente a 2.71 millones de ha) y ganaderas (3.78 millones de ha), respectivamente (Toledo y Ordóñez, 1998). Como consecuencia, el paisaje forestal original ha sido transformado en un mosaico de fragmentos de selva de diversa forma y tamaño, rodeados de áreas abiertas de aspecto sabanoide, denominadas localmente como potreros.

Los potreros son cada vez más comunes en el paisaje del trópico húmedo de México, están presentes en toda su extensión e influyen directamente sobre el aislamiento y la conectividad de los fragmentos remanentes de selva. En vastas regiones, son el elemento o componente dominante del paisaje (Guevara et al., 1997; Vitousek et al., 1997; Toledo y Ordóñez, 1998) no solamente por la extensión que ocupan, sino también por el enorme efecto que tienen sobre los procesos ecológicos y funcionamiento del ecosistema o agrosistema, que está siendo explotado (Uhl et al., 1988; Toledo, 1992; Reiners et al., 1994; Guevara et al., 1998). Sin embargo, el conocimiento sobre su estructura y composición florística es notablemente pobre, tanto en el trópico húmedo mexicano como en el resto de América, debido sobre todo a que no han sido muestreados rigurosamente para este propósito.

Sarmiento (1996) llama a los potreros neotropicales "pastizales tropicales secundarios", de acuerdo con las siguientes características: 1) son derivados de selva; 
2) suelen estar dominados en cobertura por especies de gramíneas nativas de América o introducidas de África; 3) tienen menos especies de pastos que de malezas; y 4) son inestables por tratarse de etapas serales cuyo destino dependerá sobre todo del uso al que han estado sometidos, tales como la carga animal, el tipo de pastoreo, los procedimientos de deshierbe, el uso de fertilizantes y el empleo del fuego (ver también Guevara et al., 1997).

La composición florística de los potreros en Los Tuxtlas ha sido descrita parcialmente por Purata (1986), Guevara et al. (1992, 1994), González-Montagut (1996), Williams-Linera et al. (1998), Martínez-Garza y González-Montagut (1999) y Barrera-Láez (2003). En otros lugares de México y Sudamérica se han realizado diversos estudios relacionados con la vegetación de los potreros, enfocados principalmente al efecto que esta vegetación tiene sobre el potencial de regeneración de la selva (Reiners et al., 1994; Otero-Arnaiz et al., 1999; Uhl et al., 1988; Buschbacher et al., 1988; Posada et al., 2000; Zimmerman et al., 2000; Mitchell et al., 2000). Estas publicaciones, en conjunto, dan sólo una idea parcial de la riqueza y composición florística de los potreros, pues casi sin excepción se enfocan al efecto que la deforestación y el establecimiento de campos ganaderos tienen sobre la pérdida de especies de plantas nativas de la selva.

El objetivo de este trabajo es mostrar la riqueza, la composición florística y la estructura de la vegetación de los potreros del norte de la sierra de Los Tuxtlas en los alrededores de la Estación de Biología Tropical de la Universidad Nacional Autónoma de México (EBT-UNAM), haciendo énfasis en las plantas que crecen por fuera de la sombra de los árboles que forman parte de los mismos. En particular nos interesa determinar si el tipo e intensidad de manejo influyen sobre la riqueza de la vegetación, para lo cual muestreamos en dos localidades colindantes que difieren claramente en las prácticas de manejo de sus potreros (Balzapote y La Palma). Incluimos en el estudio los dos tipos de potrero más comunes en la región: los sembrados con pasto estrella y los inducidos (sin siembra de pastos) denominados localmente "gramas" y además, hicimos levantamientos durante la época de seca y la de lluvia para considerar la variación estacional de esta vegetación.

\section{MÉTODOS}

Sitio de estudio

La sierra de Los Tuxtlas es un macizo de origen volcánico, ubicado entre los $18^{\circ} 05^{\prime}$ y $18^{\circ} 45^{\prime}$ de latitud norte y los $94^{\circ} 35^{\prime}$ y $95^{\circ} 30^{\prime}$ de longitud oeste, con orientación NO-SE, localizado al sur del estado de Veracruz. Es una sierra aislada de otros sistemas montañosos en la planicie costera del Golfo de México, con un área 
aproximada de $3,299 \mathrm{~km}^{2}$ (Geissert, 2004). La precipitación total anual en la vertiente atlántica del Volcán San Martín Tuxtla es de unos 4,000 mm, distinguiéndose (según Soto, 2004) una época seca, de marzo a mayo (precipitación promedio por mes inferior a $100 \mathrm{~mm}$ ) y una de lluvia, de junio a febrero (precipitación promedio por mes superior a $200 \mathrm{~mm}$ ). La extensión de los potreros en la sierra de Los Tuxtlas en 1991 era de 160,507 ha (Castillo y Laborde, 2004), aproximadamente 48\% de la superficie total de la sierra.

Establecimiento del potrero

El antecedente inmediato al establecimiento de un potrero es una parcela agrícola, generalmente de maíz, que con frecuencia es alternada con frijol, chile, arroz, cacahuate o piña. En la región de estudio, es común la distinción entre dos tipos de potreros: los de grama y los de estrella (Martínez-Gutiérrez, 1980; Guevara et al., 1997). El primer tipo se induce inmediatamente después de la segunda o tercera cosecha que se ha hecho en el sitio, mediante la introducción de bovinos al campo que todavía contiene las plantas de maíz pero ya sin las mazorcas. El pisoteo y forrajeo del ganado favorecen el crecimiento de pastos cuyas semillas fueron acumulándose en el banco del suelo durante la fase de milpa y son también dispersadas y traídas por estos animales desde otros potreros de grama.

Para el establecimiento de un potrero de estrella se plantan de forma manual secciones de estolón de la gramínea africana Cynodon plectostachyus Pilg., enterrándolas entre los individuos de maíz antes de cosechar las mazorcas. La entrada del ganado al sitio se realiza hasta que el pasto sembrado (vegetativamente) haya enraizado y tenga una cobertura relativamente alta (Martínez Gutiérrez, 1980; Guevara et al., 1997; Lira-Noriega, 2003). Esta especie de pasto es conocida comúnmente como estrella africana y fue introducida al trópico mexicano desde la década de 1970 (Rzedowski y Calderón, 1990).

El control de especies indeseables se hace mediante corte con machete o aspersión de herbicidas. El primero es una práctica muy común que se realiza generalmente dos veces por año y el segundo es menos frecuente porque depende de los recursos económicos del propietario. El uso del fuego no forma parte de las prácticas de manejo en los potreros de la zona estudiada. El ganado criado es una mezcla de cebú (Bos indicus) con otras razas europeas como el suizo (Bos taurus). La carga animal usual es por lo regular entre una y dos cabezas de ganado por hectárea (siendo menor la capacidad de los potreros de grama), y su rotación es muy variada, pues hay desde aquellos propietarios que mueven a su ganado diariamente hasta los que lo dejan hasta que el pasto se agota. Se calcula que la vida media de los potreros de la región es mayor que 15 años (Martínez-Gutiérrez, 1980; Guevara et al., 1997; Lira-Noriega, 2003). 
Muestreo

El muestreo se hizo en dos localidades colindantes: el ejido Balzapote y la colonia agrícola ganadera La Palma, siendo ambas colindantes con la EBT-UNAM, la primera ubicada al N y NE y la segunda al E y SE. La Palma, establecida a mediados de la década de 1930, es un asentamiento al menos diez años más antiguo que Balzapote, y ahí la ganadería ha sido la principal actividad económica desde su fundación. En el ejido Balzapote la agricultura milpera fue el aprovechamiento preponderante hasta mediados de la década de los años ochenta, época en que la desplazó la ganadería.

En cada localidad se escogieron 10 potreros: cinco de grama y cinco de estre1la (Fig. 1), se inventarió su vegetación en 1992, durante la temporada seca, del 10 de abril al 12 de mayo, y durante la de lluvia, del 25 de septiembre al 11 de octubre. Los 20 potreros se encontraban en uso durante el periodo de muestreo. El tamaño de los mismos osciló entre 2.5 y 30 ha, y su altitud sobre el nivel del mar varió entre 90 y 380 m (Cuadro 1).

En cada potrero se trazó una hectárea mediante dos ejes perpendiculares de 100 x 100 m, uno con orientación E-O (eje x) y el otro N-S (eje y), evitando en lo posible la presencia de árboles en su interior y colocando toda la hectárea dentro de una misma unidad de manejo (i.e. sin subdivisiones o cercas). Se enterraron cinco estacas que marcaron los sitios en los cuales se ubicaron los ejes (cuerdas marcadas cada $0.5 \mathrm{~m}$ ), una por cada esquina y la quinta al centro de la superficie muestreada (coordenada $\mathrm{x}, \mathrm{y}=50,50$ ). En cada hectárea se distribuyeron al azar 10 cuadros de 2 x 2 m mediante la elección aleatoria de dos números entre 0 y 98 por cada eje (coordenadas $\mathrm{x}, \mathrm{y}$ ) correspondiente a la esquina $\mathrm{SO}$ de cada cuadro. En los pocos casos en los que fue imposible evitar la presencia de árboles (principalmente naranjos) dentro de la hectárea escogida, entonces se eliminó toda combinación de números al azar que quedara a menos de $5 \mathrm{~m}$ del límite externo de la copa del árbol, garantizando que los 10 cuadros de cada potrero estuvieran ubicados a amplia distancia de la copa de cualquier árbol (sin sombra). Los mismos cuadros $(\mathrm{N}=200)$ fueron utilizados en el muestreo de ambas temporadas, teniendo una exactitud de re-localización de la época seca a la de lluvia de $\pm 0.5 \mathrm{~m}$, gracias a las estacas enterradas que marcaban la posición de los ejes.

En cada cuadro se estimó la cobertura de todas las especies de angiospermas y pteridofitas enraizadas usando los índices de Küchler (valores de 1 a $6:<1 \%, 1-5 \%$, $5-25 \%, 25-50 \%, 50-75 \%$ y $>75 \%$, respectivamente, Matteucci y Colma, 1982) y se midió la altura máxima y mínima de la vegetación. Para sumar la cobertura total por 


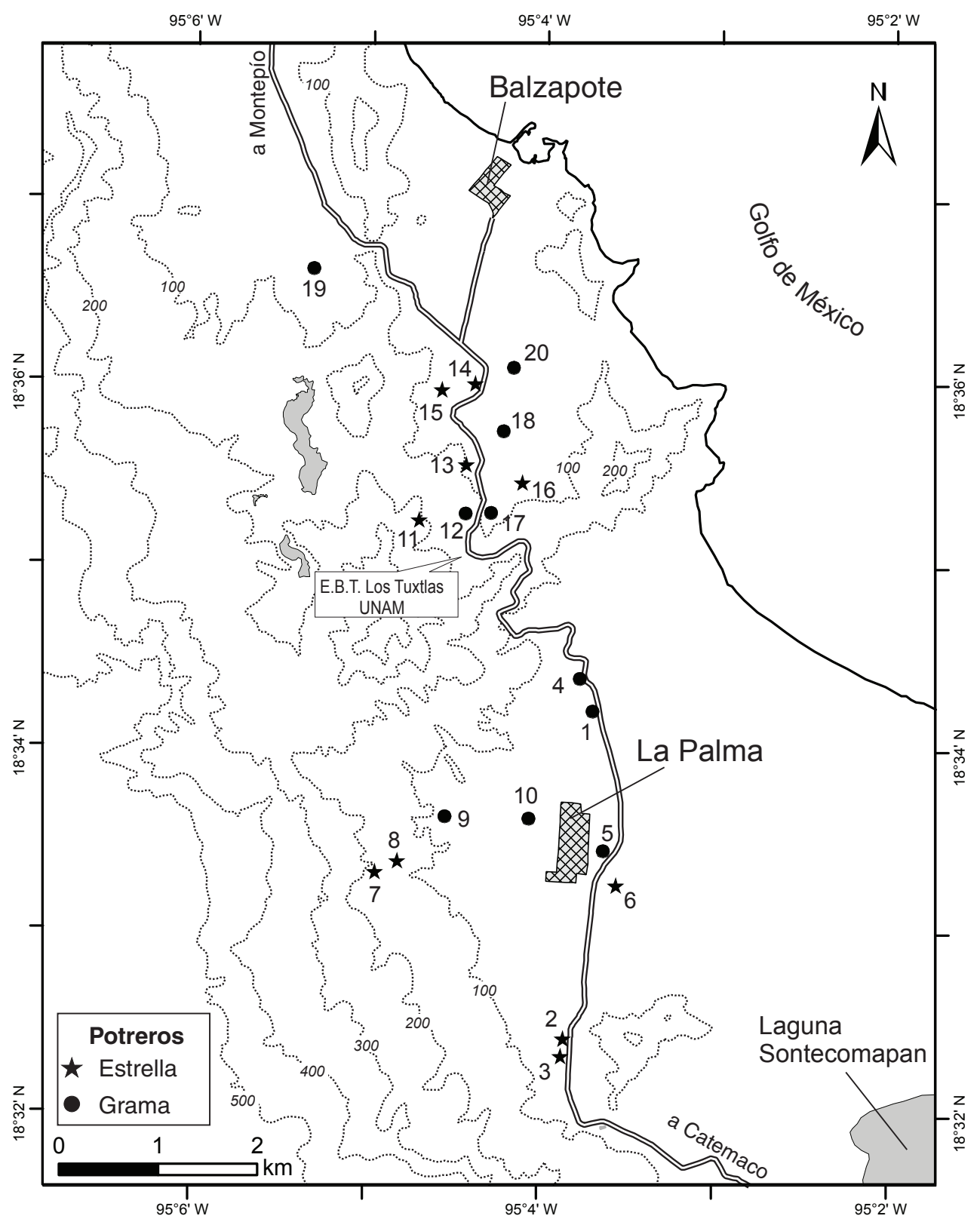

Fig. 1. Ubicación de los 20 potreros donde se llevó a cabo el muestreo en el ejido Balzapote y la colonia agrícola ganadera La Palma, al norte de la Sierra de Los Tuxtlas, Veracruz. (Basado en fotografía aérea, INEGI 1991; escala 1:75,000). 
Cuadro 1. Características ambientales y de manejo de los 20 potreros en los que se llevó a cabo el muestreo. *Indica que es información medida directamente en campo y ** indica que es información obtenida en las entrevistas realizadas a los propietarios de los potreros.

\begin{tabular}{|c|c|c|c|c|c|}
\hline Clave en Figura 1 & $\begin{array}{c}\text { Tipo de } \\
\text { potrero* }\end{array}$ & $\begin{array}{c}\text { Altitud } \\
\text { (m s.n.m)* }\end{array}$ & $\begin{array}{l}\text { Pendiente } \\
\text { (grados)* }\end{array}$ & $\begin{array}{l}\text { Edad aprox. } \\
(\text { años })^{* *}\end{array}$ & $\begin{array}{l}\text { Tamaño } \\
\text { (ha)** }\end{array}$ \\
\hline $1 \quad$ La Palma & $\mathrm{G}$ & 116 & 10.2 & - & 8 \\
\hline 2 & $\mathrm{E}$ & 166 & 0 & 15 & $\begin{array}{c}25 \text { en } 4 \\
\text { secciones }\end{array}$ \\
\hline 3 & $\mathrm{E}$ & 166 & 0 & 15 & - \\
\hline 4 & G & 132 & 16.0 & 30 & 3.5 \\
\hline 5 & G & 94 & 0 & 42 & 25 \\
\hline 6 & $\mathrm{E}$ & 108 & 0 & 24 & 25 \\
\hline 7 & $\mathrm{E}$ & 378 & 13.0 & - & - \\
\hline 8 & $\mathrm{E}$ & 316 & 13.0 & $20-25$ & $\begin{array}{c}\text { 12, más } \\
6 \text { con } \\
\text { pedrería }\end{array}$ \\
\hline 9 & G & 164 & 15.5 & 35 & 20 \\
\hline 10 & G & 100 & 0 & 30 & 8 \\
\hline 11 Balzapote & $E$ & 236 & 18.0 & 6 & 3 \\
\hline 12 & G & 192 & 19.0 & 16 & 10 \\
\hline 13 & $\mathrm{E}$ & 204 & 12.0 & 15 & 5 \\
\hline 14 & $\mathrm{E}$ & 142 & 6.6 & 20 & 12 \\
\hline 15 & $\mathrm{E}$ & 176 & 7.0 & 25 & 5 \\
\hline 16 & $\mathrm{E}$ & 136 & 3.0 & 30 & 30 \\
\hline 17 & $\mathrm{G}$ & 144 & 11.5 & 25 & 2.5 \\
\hline 18 & G & 126 & 3.0 & 30 & 8 \\
\hline 19 & G & 104 & 10.0 & $27-30$ & 15 \\
\hline 20 & $\mathrm{G}$ & 109 & 4.0 & $24-33$ & $14-15$ \\
\hline
\end{tabular}

Tipo de potrero $(\mathrm{G}=$ grama; $\mathrm{E}=$ estrella).

especie o por familia, los datos de campo (valores 1 a 6) se transformaron en el valor medio de clase (vmc) del intervalo correspondiente en metros cuadrados (ej.: valor 5 $=50-75 \%$ del cuadro; vmc $=62.5 \%=2.5 \mathrm{~m}^{2}$ de cobertura).

La identificación de especies se realizó en los herbarios XAL, Instituto de Ecología, A.C., Xalapa, Veracruz; MEXU, Universidad Nacional Autónoma de México; FCME, Facultad de Ciencias, Universidad Nacional Autónoma de México; 
y ENCB, Escuela Nacional de Ciencias Biológicas, Instituto Politécnico Nacional, con la ayuda de especialistas. Además, durante el trabajo de campo se contó con la ayuda de expertos que conocen al detalle la flora de la región para la identificación de las especies en el campo, particularmente de plántulas de árboles y arbustos. Los ejemplares correspondientes se depositaron en el Herbario XAL, y a la fecha no han sido incorporados. En todos los casos los ejemplares recogidos en el campo se tomaron de la vecindad del cuadro muestreado, nunca de su interior. Algunas especies no fueron colectadas por estar bien identificadas y referidas en el Herbario XAL o en el herbario de la EBT-UNAM. La nomenclatura del listado se basa en Brummitt y Powell (1992), Sosa y Gómez-Pompa (1994) y en Ibarra y Sinaca (1995, 1996a, 1996b). Las familias de Angiospermae se separaron en Magnoliopsida y Liliopsida (Cronquist, 1981). La asignación de la forma de crecimiento para cada especie se basó en datos de campo, de herbario y bibliográficos. Debido a que la identidad de Axonopus compressus y A. affinis no pudo definirse al momento del muestreo, se trataron en los análisis como un solo complejo que será denominado de aquí en adelante: Axonopus spp.; entendiendo que corresponde a una o ambas especies.

Análisis de datos

Se utilizó el índice de Sørensen para estimar la similitud en la composición florística entre localidades y tipos de potrero; sus valores próximos a 1 denotan que no hay mayor diferencia y los cercanos a 0 señalan una mínima semejanza, debida a un alto recambio espacial de las especies (Matteucci y Colma, 1982; Moreno, 2001).

Las diferencias en la riqueza promedio de especies por cuadro $(2 \times 2 \mathrm{~m})$ en cada temporada de muestreo por separado (seca y lluvia), se analizaron mediante un ANDEVA de dos vías para determinar el efecto de la localidad (Balzapote vs. La Palma) y el tipo de potrero (grama vs. estrella) sobre la riqueza de la vegetación. Mediante la prueba de Anderson-Darling (Dytham, 1999) se determinó que la riqueza por cuadro no difirió de una distribución normal en ninguna de las dos temporadas y por ende no hubo necesidad de transformar dicha variable para realizar el ANDEVA.

Para evaluar la representatividad del muestreo se comparó la acumulación de especies observada con el modelo paramétrico de Clench y los no paramétricos de $\mathrm{Jack}_{1}$ y Chao $_{2}$ (Moreno, 2001). Las muestras se ordenaron de forma aleatoria con el programa EstimateS v. 7.5 (Colwell, 2005) con el fin de eliminar la arbitrariedad con que pueden añadirse, y de ese modo alterar la forma de la curva de acumulación de 
especies. Los valores de los estimadores no paramétricos de la riqueza específica se obtuvieron con el mismo programa. El ajuste del modelo paramétrico se hizo en el programa SigmaStat v. 2.03 (SPSS Inc., 1997).

\section{RESULTADOS}

Se registraron 214 especies de plantas vasculares, pertenecientes a 57 familias; 200 angiospermas y 14 pteridofitas. De este total, hubo 15 para las cuales no logramos determinar la especie pero sí el género y otras 9 para las cuales no logramos determinar el género pero sí la familia. Todas las especies se enlistan en el Apéndice, con su forma de crecimiento, localidad y tipo de potrero en que se encontraron.

Las familias con mayor número de especies fueron Asteraceae (26), Poaceae (24), Leguminosae (20, incluyendo: 5 Caesalpiniaceae, 12 Fabaceae y 3 Mimosaceae), Euphorbiaceae (16), Rubiaceae (9), Cyperaceae (7), Solanaceae (7) y Thelypteridaceae (7). Las familias con mayor cobertura fueron Poaceae $(63 \%$ de la superficie muestreada), Lamiaceae (20\%), Leguminosae (19\%) y Cyperaceae $(7 \%)$. La proporción de la cobertura de cada familia entre temporadas se mantuvo constante.

Agrupadas por su forma de crecimiento, las plantas mejor representadas fueron las hierbas con 152 especies, superando en órdenes de magnitud a la riqueza de las demás categorías (Fig. 2). Destaca la presencia de 19 especies de arbustos y 17 de árboles (ver Apéndice), registradas todas como plántulas.

El número total de especies inventariadas en Balzapote fue 188 y en La Palma 131. En conjunto, en los potreros de grama hubo un total de 162 y en los de estre1la 165. En la temporada seca se detectaron 171 y en la de lluvia 172. El índice de Sørensen indica que hay una relativamente alta similitud en la composición florística tanto en temporada seca como en la de lluvia entre las localidades (Balzapote vs. La Palma: 0.65 en ambas épocas) y entre los tipos de potrero (grama vs. estrella: 0.70 en la primera y 0.65 en la segunda). En la estación seca la altura máxima de la vegetación fue de $33.7 \pm 1.0 \mathrm{~cm}$ y la mínima de $7.1 \pm 0.6 \mathrm{~cm}$, mientras que en la de lluvia la máxima fue de $53.8 \pm 1.1 \mathrm{~cm}$ y la mínima de $15.8 \pm 0.9 \mathrm{~cm}$.

Las especies de mayor frecuencia no siempre fueron las de mayor cobertura y para algunas registramos cambios notables en esta última entre las muestras de las dos temporadas (Fig. 3). Solamente once especies superaron los $20 \mathrm{~m}^{2}$ de cobertura ( $>2.5 \%$ de la superficie inventariada) durante la temporada seca (Fig. 3a) y únicamente ocho durante la de lluvias (Fig. 3b). Solamente diez fueron encontradas en más de $60 \%$ de los 200 cuadros (i.e. ampliamente distribuidas), entre ellas, cinco 


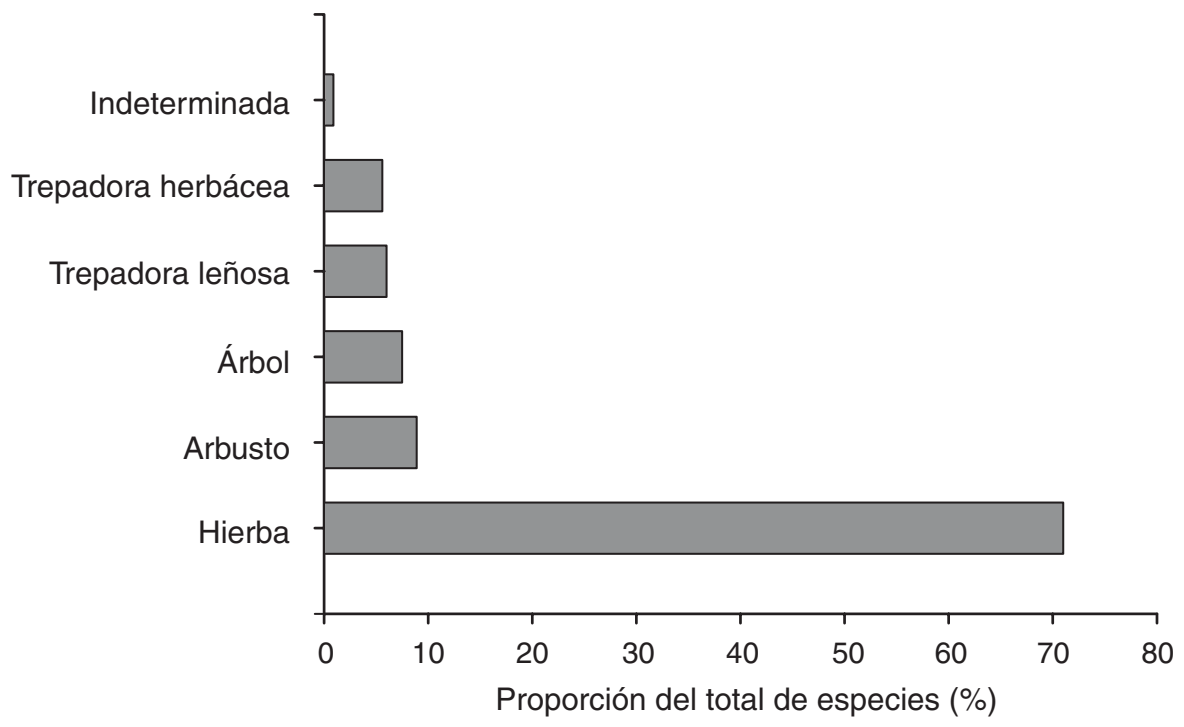

Fig. 2. Espectro de las proporciones de las diferentes formas de crecimiento de las especies registradas en el muestreo de los 20 potreros.

tuvieron una cobertura total relativamente baja (menor a $40 \mathrm{~m}^{2} ; 5 \%$ de la superficie muestreada) durante ambas temporadas: Kyllinga breviflora (Cyperaceae), Sida rhombifolia (Malvaceae), Rhynchospora radicans (Cyperaceae), Phyllanthus urinaria (Euphorbiaceae) y Borreria laevis (Rubiaceae). Las restantes cinco de muy amplia distribución alcanzaron valores de cobertura notablemente mayores, tres de ellas tuvieron una notoria disminución en su cobertura de la temporada seca a la de lluvia: Hyptis atrorubens (Lamiaceae), Axonopus spp. y Paspalum conjugatum (Poaceae), mientras que las dos restantes: C. plectostachyus (Poaceae) y Mimosa pudica (Mimosaceae) aumentaron su cobertura durante las lluvias.

Durante la temporada de secas, tanto la localidad $\left(\mathrm{F}_{(1,196)}=35.72 ; \mathrm{p}<0.001\right)$ como el tipo de pasto $\left(\mathrm{F}_{(1,196)}=20.60 ; \mathrm{p}<0.001\right)$ y la interacción de ambos factores $\left(\mathrm{F}_{(1,196)}=7.73 ; \mathrm{p}<0.001\right)$ tuvieron un efecto significativo en la riqueza promedio por cuadro $\left(4 \mathrm{~m}^{2}\right)$; siendo ésta considerablemente mayor en los potreros de grama de Balzapote que en cualquiera de las otras tres combinaciones estudiadas (Fig. 4a). Durante la estación de lluvias, el efecto de la localidad $\left(\mathrm{F}_{(1,196)}=29.35 ; \mathrm{p}<0.001\right)$ y del tipo de pasto $\left(\mathrm{F}_{(1,196)}=23.80 ; \mathrm{p}<0.001\right)$ sobre la riqueza, siguieron siendo significativos (Fig. 4b), sin embargo, durante esta época la interacción de ambos factores no fue significativa $\left(\mathrm{F}_{(1,196)}=2.19 ; \mathrm{p}=0.140\right)$. En ambas temporadas los potreros de estrella de La Palma fueron los más pobres con un promedio menor de 16 especies por cuadro y 

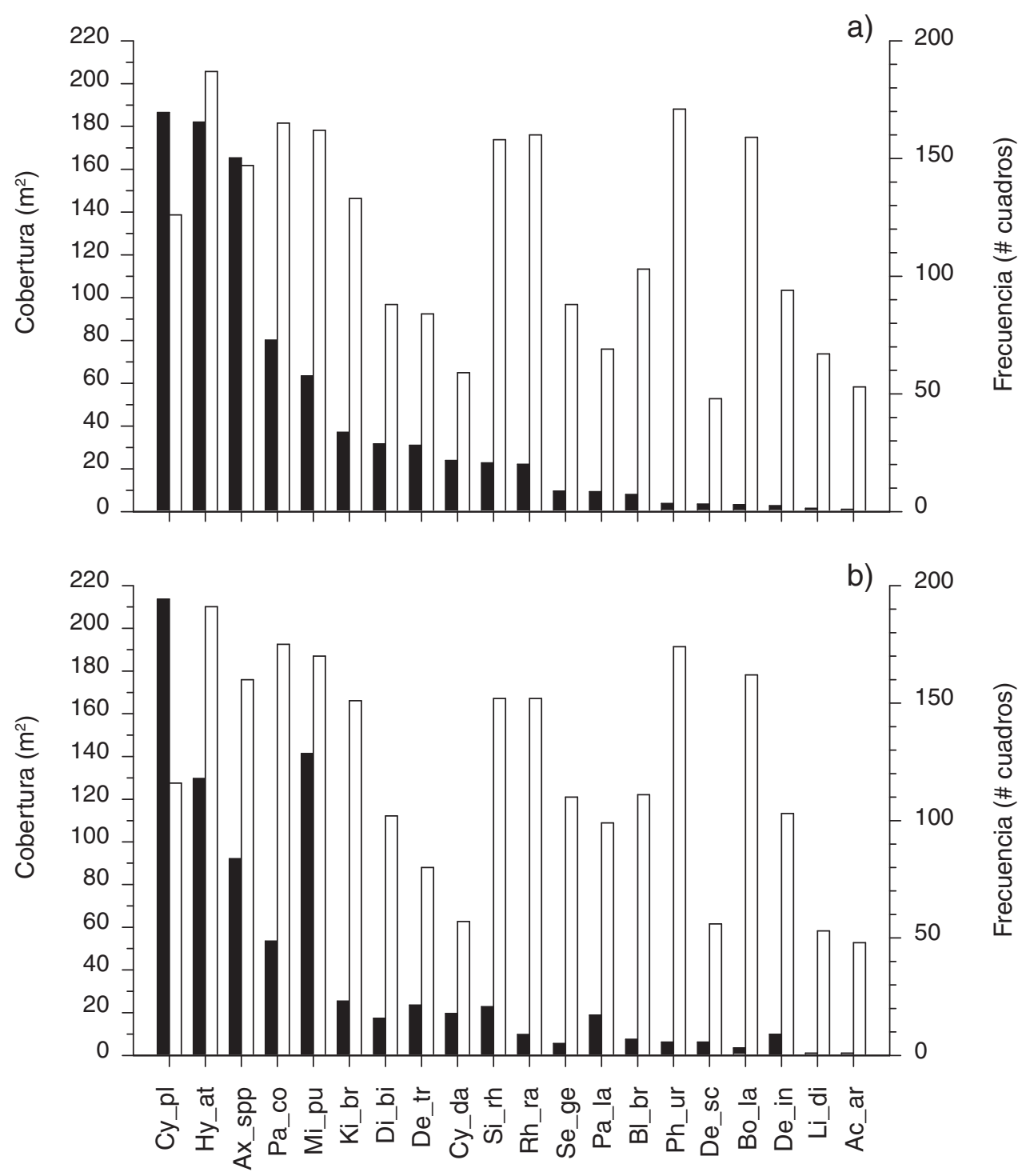

Fig. 3. Comparación cuantitativa de las especies cuya frecuencia relativa (barras blancas) fue igual o mayor de $24 \%$ (aparecieron en 48 o más cuadros) durante ambas temporadas en los 20 potreros, mostrando también su cobertura en metros cuadrados (barras negras) durante la temporada seca (a) y de lluvia (b). Axonopus compressus y A. affinis se consideran como una sola entidad por ser un complejo no distinguible en campo. El nombre de cada especie se indica con las dos primeras letras del género y de la especie. 


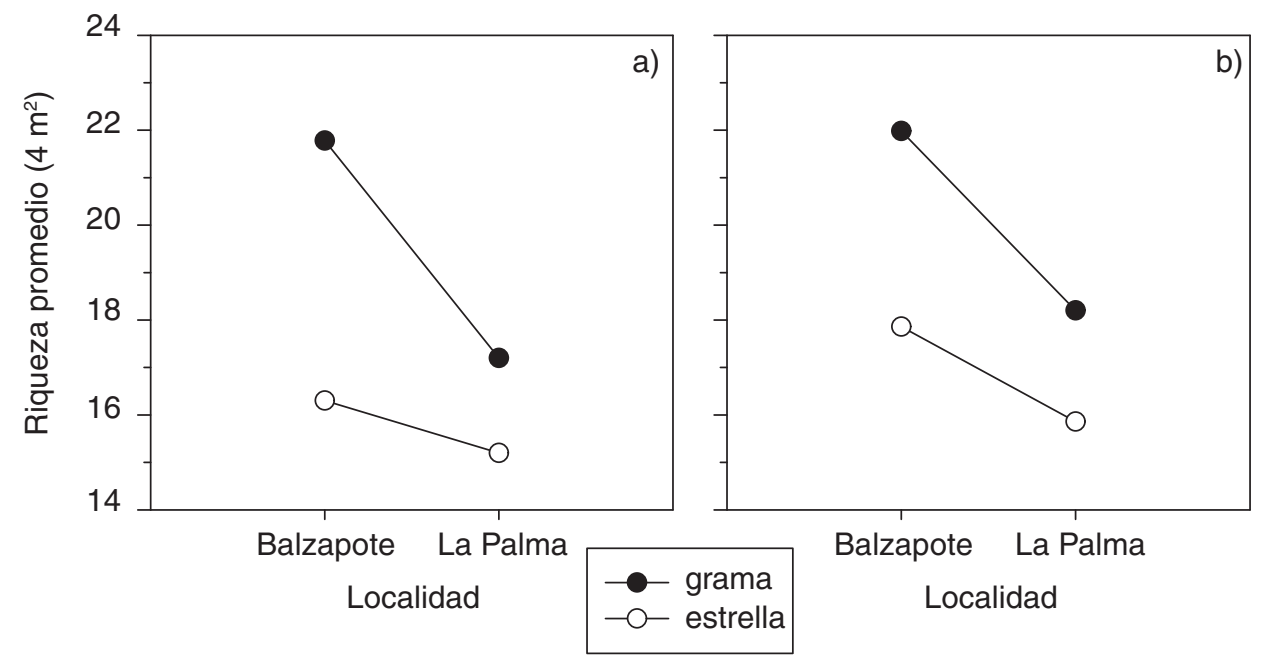

Fig. 4. Gráficas que muestran la variación de la riqueza promedio por cuadro de $4 \mathrm{~m}^{2}$ en función de la localidad (Balzapote y La Palma) y el tipo de pasto (grama y estrella), durante la temporada seca (a) y de lluvia (b).

los de grama de Balzapote fueron los más ricos con un promedio cercano a 22 (Fig. $4 a$ y $4 b)$.

Representatividad del muestreo

La tasa de acumulación de especies observada indica que el muestreo fue suficiente para disminuir considerablemente la probabilidad de aparición de nuevas plantas, por lo que representa una buena aproximación de la riqueza total del sistema estudiado. El modelo de acumulación de Clench calcula un máximo de 189 y 193 especies en temporada de seca y de lluvia, respectivamente, es decir de 18 a 21 más de las registradas por temporada, si ampliamos considerablemente la superficie de muestreo. Por otro lado, los estimadores no-paramétricos de la riqueza específica, mismos que se basan en la proporción y tasa de aparición de plantas raras, revelan una diversidad potencial mayor a la registrada. El estimador no-paramétrico de tipo "jacknife" de primer orden ( $\left.\mathrm{Jack}_{1}\right)$ indica un máximo de 218 y 223 especies en temporada seca y de lluvia, respectivamente, mientras que el de tipo "Chao" de segundo orden $\left(\mathrm{ChaO}_{2}\right)$ arroja un máximo de 224 y 254 para los mismos casos. Es decir, estos estimadores no-paramétricos de la riqueza revelan que se esperaría encontrar entre $47\left(\mathrm{Jack}_{1}\right)$ y $82\left(\mathrm{ChaO}_{2}\right)$ especies más de las registradas (Fig. 5), sin embargo se trataría en todos los casos de elementos de muy baja frecuencia. 
Lira-Noriega, et al.: Composición florística de potreros de Los Tuxtlas, Veracruz
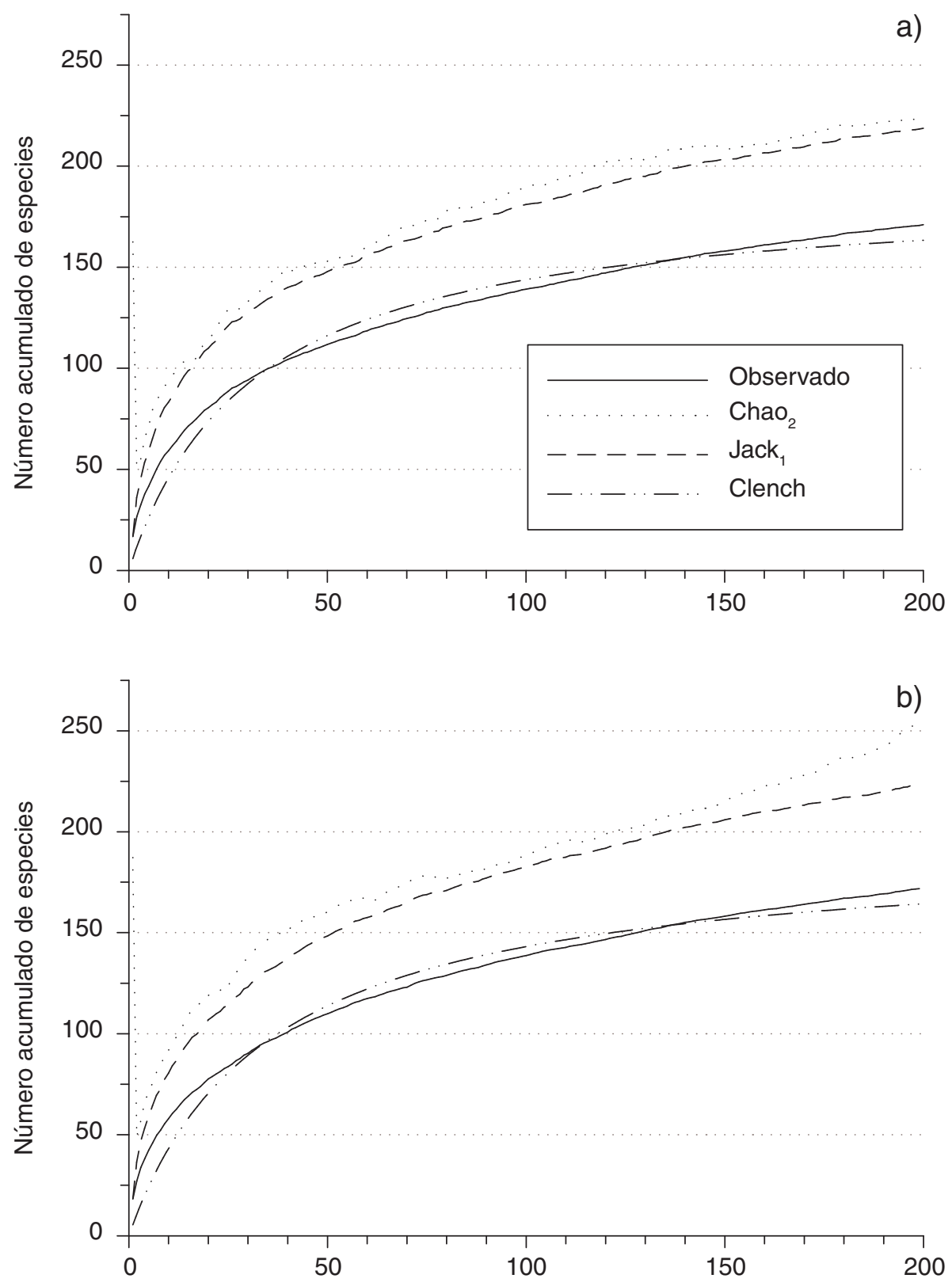

Fig. 5. Curva de acumulación de especies observada, así como las predichas por el modelo paramétrico de Clench y los modelos no paramétricos de tipo "jacknife" de primer orden $\left(\mathrm{Jack}_{1}\right)$ y de tipo "Chao" de segundo orden $\left(\mathrm{Chao}_{2}\right)$, para valorar la representatividad del muestreo en los 20 potreros, durante la temporada seca (a) y de lluvia (b). 


\section{DISCUSIÓN}

Los potreros de la vertiente atlántica (del Golfo) del volcán San Martín Tuxtla, en los alrededores de la EBT-UNAM, son ricos en especies de plantas y su composición y riqueza varían en relación con el tipo e intensidad de manejo que se emplea en ellos. Algunas de las variables que afectan las características de esta vegetación son: el procedimiento de establecimiento de los pastos (i.e. siembra vs. inducción), la frecuencia de aspersión de herbicidas y desmalezamiento con machete (chapeo), la carga animal (número de cabezas de ganado / superficie) y otras relacionadas con el uso anterior de cada predio, desde el momento de la tala de la selva original. Tales factores suelen estar íntimamente asociados con la historia de la localidad en la que se encuentran (Lira-Noriega, 2003). En pastizales de clima templado se ha comprobado que la carga animal, la alternancia y duración de los periodos de pastoreo y descanso, así como la intensidad y frecuencia de las prácticas de aclareo o deshierbe, son los elementos que mas influyen en la riqueza de plantas. En dichos pastizales particularmente en Europa, las prácticas tradicionales de manejo que durante varios siglos se han afinado para optimizar el rendimiento pecuario y mantenimiento del forraje, también maximizan la diversidad de plantas (Bárbaro et al., 2001; Olff y Ritchie, 1998; Posada et al., 2000).

La variabilidad de la composición florística de los potreros de Los Tuxtlas se manifiesta en función de la proporción de especies raras o de baja frecuencia, mismas que en gran parte explican las diferencias florísticas de la vegetación detectadas entre las localidades estudiadas y entre los tipos de potrero (grama o estrella). Del total de 214 especies registradas, $61(28 \%)$ aparecieron solamente una vez (en un cuadro de muestreo) y 39 (18\%) entre dos y diez veces.

Como era de esperarse, en las zonas totalmente abiertas del potrero predominan las hierbas, sin embargo, llama la atención también la presencia de otras formas de crecimiento, particularmente la de arbustos y árboles, registrados principalmente como plántulas. Las 35 especies de estas categorías son componentes de la selva y acahuales de la zona, hecho que puede ser importante para la regeneración de la vegetación leñosa en los potreros.

Entre los elementos de mayor cobertura destacan las gramíneas de crecimiento por estolones (Cynodon plectostachyus, Axonopus compressus, A. affinis, Paspalum notatum, $P$. conjugatum), lo que al parecer les permite cubrir áreas amplias y resistir el forrajeo del ganado, así como los cambios en la estacionalidad anual (McIlroy, 1973; Bogdan, 1977). Otras plantas frecuentes y de amplia cobertura son poco palatables (Hyptis atrorubens) o tienen espinas como defensa (Mimosa pudica) resaltando la importancia de tales formas de protección. 
Cabe destacar también que algunas especies de talla muy pequeña o capaces de producir semillas con individuos muy chicos y en muy corto tiempo, tales como Kyllinga breviflora, Rhynchospora radicans, Sida rhombifolia, Phyllanthus urinaria y Borreria laevis, son capaces de mantenerse en los potreros con abundancias y coberturas relativamente bajas pero con una distribución muy amplia (valores de frecuencia muy altos), seguramente debido a que su reducida talla les permite escapar al forrajeo del ganado y a que sus semillas forman parte del banco del suelo de los potreros (Guevara et al., 2004; 2005), lo que a su vez les permite escapar temporalmente de condiciones adversas.

La altura de la vegetación estudiada (mínima de $7 \mathrm{~cm}$ y máxima de $54 \mathrm{~cm}$ ) se aproxima a la que otros autores reconocen como óptima o deseable para el pastoreo por bovinos en praderas tropicales (McIlroy, 1973). Una baja estatura de la cubierta vegetal debida al forrajeo del ganado puede corresponder a una mayor riqueza florística en concordancia con la hipótesis de la complejidad estructural y del forrajeo medio, de acuerdo con la cual se espera que las especies dominantes y agresivas que sean palatables se mantengan con coberturas relativamente reducidas y permitan mayor diversidad de plantas (Hobbs y Huenneke, 1992; Olff y Ritchie, 1998). En los potreros muestreados están bien representadas las familias que tienen especies poco palatables y en ocasiones tóxicas para el ganado, como Asteraceae, Euphorbiaceae y Solanaceae (Harvard-Duclos, 1975), al mismo tiempo que también encontramos elementos altamente preferibles por los bovinos (i.e. mayoría de los pastos). La intrincada mezcla de plantas palatables y no palatables afecta directamente a la ubicación del forrajeo en el terreno y a la riqueza local a nivel de parche.

La riqueza promedio por cuadro $\left(4 \mathrm{~m}^{2}\right)$ en este muestreo, fue considerablemente mayor en los potreros de grama de Balzapote, seguidos por los potreros de grama de La Palma, luego los de estrella de Balzapote y por último los de estrella de La Palma. Tal orden sugiere que el método de establecimiento del potrero así como la historia de uso del mismo, son muy importantes para entender la variación de la riqueza entre sitios. Este resultado concuerda con el hecho de que la localidad de Balzapote es un ejido que adoptó a la ganadería como modo de subsistencia de manera secundaria a la agricultura, debido a lo cual sus terrenos han tenido más ciclos de roza-tumba-quema y de rotación de cultivos que en La Palma. Lo anterior resulta en una notable acumulación de numerosas especies de malezas que persisten o mantienen altas densidades en los potreros (Guevara et al., 2005). La colonia agrícola ganadera La Palma se funda con gente de mayor capacidad técnica y monetaria para la ganadería y en ella se establecen los potreros de manera más directa, sin pasar por varios ciclos agrícolas (Lira-Noriega, 2003). Además, habría que enfa- 
tizar que fue en los potreros de estrella de La Palma, en los que la riqueza local o de parche fue consistentemente menor en ambas temporadas de muestreo, con tres a cinco especies menos por cuadro en comparación con las otras tres condiciones. Dichos potreros son en los que mejor se cuida el pasto sembrado (i.e. estrella), principalmente mediante un minucioso control de la carga y rotación animal así como con el empleo más frecuente y extendido de herbicidas que eliminan dicotiledóneas selectivamente, favoreciendo el crecimiento de las monocotiledóneas, en particular aquellas que son más resistentes al pastoreo y pisoteo del ganado, tales como los pastos, cuyo denso follaje excluye localmente a otras especies.

Con respecto a los listados florísticos para la EBT-UNAM "Los Tuxtlas" de Ibarra y Sinaca (1995, 1996a, 1996b), encontramos nuevos registros en 14 familias (ver Apéndice), entre las que destacan Poaceae con 17 especies que no habían sido previamente citadas para la zona, Asteraceae con 9 y Euphorbiaceae con 4. Este es un componente importante de la flora que se encuentra en las zonas abiertas (por fuera de la selva) y que contribuyen a la riqueza florística de la región.

En las mismas localidades muestreadas en este estudio, que colindan con la EBT-UNAM, se han hecho diferentes muestreos del arbolado que encontramos en sus potreros (Guevara et al., 1997; 1998; Barrera-Láez, 2003; González-Montagut, 1996) o de la vegetación asociada con los árboles de los potreros o su sombra (Guevara et al., 1992, 1994, 2004; Williams-Linera, 1998). Si consideramos como parte del potrero al arbolado, así como a lo que crece bajo sus copas y no únicamente al pastizal abierto, entonces la flora de los potreros es mucho más rica en especies de plantas de lo que generalmente se asume. Si a las 214 especies que encontramos en este trabajo le añadimos aquéllas adicionales (principalmente arbustos y árboles) de los trabajos mencionados anteriormente, el listado florístico asciende a 480 especies pertenecientes a 94 familias. Tal riqueza de plantas corresponde únicamente a dos localidades vecinas a la reserva de selva húmeda de la UNAM y en la que la transformación del paisaje ha sido severa y prolongada, extendiéndose durante varias décadas de uso agrícola y ganadero y en las que actualmente los potreros ocupan casi la totalidad de la extensión de ambos sitios.

Una apreciación acertada de los potreros que ocupan áreas anteriormente cubiertas por selvas húmedas es la que hace Sarmiento $(1984,1996)$, quien sugiere que su referente más cercano está en los pastizales naturales y en las sabanas neotropicales, formaciones naturales que son superadas en riqueza florística por los potreros debido a la composición y alta diversidad de la selva original, así como a los procedimientos de manejo y utilización de los mismos, incluyendo las modalidades y antigüedad de la deforestación (Sarmiento, 1996). Habría que enfatizar también que 
la presencia de árboles aislados así como la vecindad de remanentes de selva, son factores que contribuyen sustancialmente a la variedad de las plantas de los potreros (Guevara et al., 1992, 1994, 2004). En realidad, los potreros de Los Tuxtlas y en general los del trópico húmedo americano son arbolados, siendo muy comunes en ellos los árboles aislados, cercas vivas y franjas riparias (Guevara et al., 1997, 1998). Tal condición coincide además con la definición del Diccionario de la Lengua Española de lo que es un potrero: "Finca rústica, cercada y con árboles, destinada principalmente a la cría y sostenimiento de toda especie de ganado (Anónimo, 1970)", que es sobre todo aceptada y frecuentemente usada en países de América.

Es importante considerar que la conversión de la selva en terrenos de uso pecuario no necesariamente resulta en una drástica disminución de la riqueza de plantas. La modificación estructural (forestal a herbácea) y florística sí son manifiestamente radicales y no podemos dejar de enfatizar que la expansión ganadera provoca una cuantiosa y lamentable disminución del tamaño poblacional de numerosas especies nativas de la selva, que está empobreciendo peligrosamente a la biodiversidad del trópico húmedo. Sin embargo, en los potreros estas plantas son sustituidas por malezas y otros elementos herbáceos de los que en su gran mayoría no sabemos de dónde vinieron, cómo llegaron, ni cómo se reproducen o mantienen ahí. La heterogeneidad espacial en riqueza y composición florística detectada en este trabajo está relacionada con atributos diversos de la historia de vida de las plantas, entre los que destacan la forma de crecimiento, la palatabilidad y capacidad de formar bancos de semillas en el suelo, atributos que en combinación con las variables de los métodos de establecimiento del potrero así como del manejo y movimiento del ganado, deberán estudiarse a fondo para comprender sus características estructurales y florísticas, así como la dinámica de la vegetación de los campos ganaderos.

\section{AGRADECIMIENTOS}

A Doris Liesenfeld, Santiago Sinaca, Otilio Barrera, Julián Temich y Santos Sinaca se agradece su participación en la realización del muestreo; así como a los especialistas que ayudaron en la identificación de ejemplares colectados: Ma. Teresa Mejía-Saulés y Jesús Valdés (Poaceae), Leticia Cabrera y Gonzalo Castillo (Compositae), Mónica Palacios (Pteridophyta); Nelly Diego (Cyperaceae), Jaime Jiménez (Euphorbiaceae); Magdalena Peña (Orchidaceae); Isidro Méndez Larios (Scrophulariaceae). Para la identificación en el campo se contó con la colaboración de Santiago Sinaca. Victoria Sosa revisó el listado de especies. La contribución de dos revisores 
anónimos ayudó a mejorar la calidad del manuscrito. Los comentarios de Claudia Moreno y Patricia Koleff ayudaron a aclarar la parte de diversidad. Este trabajo fue financiado por el Consejo Nacional de Ciencia y Tecnología (Proyecto 0239-N9107 "Diagnóstico y Conservación de la Biodiversidad en México"), y por una beca tesis del Instituto de Ecología, A. C., del proyecto Ecología Vegetal No. 902-17.

\section{LITERATURA CITADA}

Anónimo. 1970. Diccionario de la lengua española. Real Academia Española. Espasa-Calpe, S.A. Madrid. 1424 pp.

Bárbaro, L., T. Dutoit y P. Cozic. 2001. A six-year experimental restoration of biodiversity by shrub-clearing and grazing in calcareous grasslands of the French Prealps. Biodivers. Conserv. 10: 119-135.

Barrera-Láez, O. 2003. Uso y manejo de árboles en potreros de Los Tuxtlas, Veracruz. Tesis profesional. Facultad de Ciencias Agrícolas, Universidad Veracruzana. Xalapa, Veracruz. $80 \mathrm{pp}$.

Bogdan, A. V. 1977. Tropical pasture and fodder plants. Longman. Londres. 475 pp.

Brummitt, R. K. y C. E. Powell (eds.). 1992. Authors of plant names. Royal Botanic Gardens. Kew. $731 \mathrm{pp}$.

Buschbacher, R., C. Hull y E. A. S. Serrão. 1988. Abandoned pastures in eastern Amazonia. II. Nutrient stocks in the soil and vegetation. J. Ecol. 76: 682-699.

Castillo, G. y J. Laborde. 2004. La vegetación. In: Guevara, S., J. Laborde y G. SánchezRíos (eds.). Los Tuxtlas: el paisaje de la sierra. Instituto de Ecología, A.C. y Unión Europea. Xalapa. pp. 231-265.

Colwell, R. K. 2005. EstimateS: Statistical estimation of species richness and shared species from samples. Versión 7.5 User's guide and application published at: http://purl.oclc. org/estimates.

Cronquist, A. 1981. An integrated system of classification of flowering plants. Columbia University Press. New York. 1262 pp.

Dytham, C. 1999. Choosing and using statistics. A biologist's guide. Blackwell Science. Oxford. $218 \mathrm{pp}$.

Geissert, D. K. 2004. La geomorfología. In: Guevara, S., J. Laborde y G. Sánchez-Ríos (eds.). Los Tuxtlas: el paisaje de la sierra. Instituto de Ecología, A.C. y Unión Europea. Xalapa. pp. 159-179.

González-Montagut, R. 1996. Establishment of three rain forest species along the riparian corridor-pasture gradient in Los Tuxtlas, Mexico. Tesis doctoral. Harvard University. Cambridge. 503 pp.

Guevara, S., J. Meave, P. Moreno-Casasola y J. Laborde. 1992. Floristic composition and structure of vegetation under isolated trees in neotropical pastures. J. Veg. Sci. 3: 655-664. 
Guevara, S., J. Meave, P. Moreno-Casasola, J. Laborde y S. Castillo. 1994. Vegetación y flora de potreros en la Sierra de Los Tuxtlas, México. Acta Bot. Mex. 28: 1-27.

Guevara, S., J. Laborde, D. Liesenfeld y O. Barrera. 1997. Potreros y ganadería. In: GonzálezSoriano, E., R. Dirzo y R. C. Vogt (eds.). Historia natural de Los Tuxtlas. Instituto de Biología, Universidad Nacional Autónoma de México. México, D.F. pp. 43-58.

Guevara, S., J. Laborde y G. Sánchez. 1998. Are isolated remnant trees in pastures a fragmented canopy?. Selbyana 19: 34-43.

Guevara, S., J. Laborde y G. Sánchez-Ríos. 2004. Rain forest regeneration beneath the canopy of fig trees isolated in pastures of Los Tuxtlas, Mexico. Biotropica 36: 99108.

Guevara, S., P. Moreno-Casasola y G. Sánchez-Rios. 2005. Soil seed banks in the tropical agricultural fields of Los Tuxtlas, Mexico. Tropical Ecology 46: 217-225.

Harvard-Duclos, B. 1975. Las plantas forrajeras tropicales: técnicas agrícolas y producciones tropicales. Blume. Barcelona. 380 pp.

Hobbs, R. J. y L. F. Huenneke. 1992. Disturbance, diversity, and invasion: implications for conservation. Cons. Biol. 6: 324-337.

Ibarra-Manríquez, G. y S. Sinaca. 1995. Lista florística comentada de la Estación de Biología Tropical "Los Tuxtlas", Veracruz. México. Rev. Biol. Trop. 43: 75-115.

Ibarra-Manríquez, G. y S. Sinaca. 1996a. Estación de Biología Tropical "Los Tuxtlas”, Veracruz, México: Lista florística comentada (Mimosaceae a Verbenaceae). Rev. Biol. Trop. 44: 41-60.

Ibarra-Manríquez, G. y S. Sinaca. 1996b. Lista comentada de plantas de la Estación de Biología Tropical "Los Tuxtlas”, Veracruz, México: (Violaceae-Zingiberaceae). Rev. Biol. Trop. 44: 427-447.

Lira-Noriega, A. 2003. La vegetación de los potreros del norte de la sierra de Los Tuxtlas. Tesis profesional. Facultad de Ciencias, Universidad Nacional Autónoma de México. México, D.F. 98 pp.

Martínez-Garza, C. y R. González-Montagut. 1999. Seed rain from forest fragments into tropical pastures in Los Tuxtlas, Mexico. Plant Ecol. 145: 255-265.

Martínez Gutiérrez, J. 1980. Prácticas tradicionales de establecimiento y uso de los potreros en una región cálido-húmeda (Balzapote, Veracruz). Tesis profesional. Facultad de Ciencias, Universidad Nacional Autónoma de México. México, D.F. 73 pp.

Matteucci, S. D. y A. Colma. 1982. Metodología para el estudio de la vegetación. Secretaría General de la Organización de Estados Americanos. Washington, D.C. 168 pp.

McIlroy, R. J. 1973. Introducción al cultivo de los pastos tropicales. Limusa. México, D.F. $168 \mathrm{pp}$.

Mitchell, A. T., J. K. Zimmerman, J. B. Pascarella, L. Rivera y H. Marcano-Vega. 2000. Forest regeneration in a chronosequence of tropical abandoned pastures: implications for reforestation ecology. Restor. Ecol. 8: 328-338.

Moreno, C. E. 2001. Manual de métodos para medir la biodiversidad. Textos Universitarios. Universidad Veracruzana. Xalapa. 49 pp.

Olff, H. y M. E. Ritchie. 1998. Effects of herbivores on grassland plant diversity. Trends Ecol. Evol. 13: 261-265. 
Otero-Arnaiz, A., S. Castillo, J. Meave y G. Ibarra-Manríquez. 1999. Isolated pasture trees and the vegetation under their canopies in the Chiapas coastal plain, Mexico. Biotropica 31: 243-254.

Posada, J. M., T. Mitchell Aide y J. Cavelier. 2000. Cattle and weedy shrubs as restoration tools of tropical montane rainforest. Restor. Ecol. 8: 370-379.

Purata, S. E. 1986. Studies on secondary succession in Mexican tropical rain forest. Tesis doctoral. Faculty of Science, Uppsala University. Uppsala, Suecia. 130 pp.

Reiners, W. A., A. F. Bouwman, W. F. J. Parsons y M. Keller. 1994. Tropical rain forest conversion to pasture: changes in vegetation and soil properties. Ecol. Appl. 4: 363377.

Rzedowski, J. y G. Calderón. 1990. Nota sobre el elemento africano en la flora adventicia de México. Acta Bot. Mex. 12: 21-24.

Sarmiento, G. 1984. The ecology of neotropical savannas. Harvard University Press. Cambridge, Massachusetts. 235 pp.

Sarmiento, G. 1996. Ecología de pastizales y sabanas en América Latina. In: Sarmiento, G. y M. Cabido (eds.). Biodiversidad y funcionamiento de pastizales y sabanas en América Latina. Programa Iberoamericano de Ciencia y Tecnología para el Desarrollo y Centro de Investigaciones Ecológicas de los Andes Tropicales. Mérida, Venezuela. pp. 15-24.

Sosa, V. y A. Gómez-Pompa (comp.). 1994. Flora de Veracruz. Lista florística. Fascículo 82. Instituto de Ecología, A.C. Xalapa. 245 pp.

Soto, M. 2004. El clima. In: Guevara, S., J. Laborde y G. Sánchez-Ríos (eds.). Los Tuxtlas: el paisaje de la sierra. Instituto de Ecología, A.C. y Unión Europea. Xalapa. pp. 195198.

SPSS Inc.1997. SigmaStat for Windows Ver. 2.03. Chicago.

Toledo, V. M. 1992. Bio-economic costs. In: Downing, T. E., S. B. Hecht, H. A. Pearson y C. Garcia-Downing (eds.). Development or destruction. Development or destruction: the conversion of tropical forest to pasture in Latin America. Westview Press. Boulder. pp. 67-94.

Toledo, V. M. y M. J. Ordóñez. 1998. El panorama de la biodiversidad de México: una revisión de los hábitats terrestres. In: Ramamoorthy, T. P., R. Bye, A. Lot y J. Fa (comp.). Diversidad biológica de México: orígenes y distribución. Instituto de Biología, Universidad Nacional Autónoma de México. México, D.F. pp. 739-757.

Uhl, C., R. Buschbacher y E. A. S. Serrão. 1988. Abandoned pastures in eastern Amazonia. I. Patterns of plant succession. J. Ecol. 76: 663-681.

Vitousek, P. M., H. A. Mooney, J. Lubchenco y J. M. Melillo. 1997. Human domination of earth's ecosystems. Science 277: 494-499.

Williams-Linera, G., V. Domínguez-Gastelú y M. E. García-Zurita. 1998. Microenvironment and floristics of different edges in a fragmented tropical rainforest. Conserv. Biol. 12: 1091-1102.

Zimmerman, J. K., J. B. Pascarella y T. Mitchell Aide. 2000. Barriers to forest regeneration in an abandoned pasture in Puerto Rico. Restor. Ecol. 8: 350-360.

Recibido en mayo de 2004.

Aceptado en abril de 2007. 
Apéndice. Lista de especies de plantas vasculares de 20 potreros del norte de la Sierra de Los Tuxtlas, Veracruz, en áreas desprovistas de árboles. Se marcan con * las especies no mencionadas en el listado florístico de Ibarra-Manríquez y Sinaca (1995, 1996a, 1996b) y con las especies de vegetación secundaria que sí fueron mencionadas en los mismos trabajos de Ibarra-Manríquez y Sinaca. Las morfoespecies cuyo género está en duda, como se indica entre paréntesis, se señalan con $\dagger$. Localidad: $\mathrm{B}=$ Balzapote; LP = La Palma. Tipo de potrero: $\mathrm{G}=$ potrero grama; $\mathrm{E}=$ potrero de estrella. Forma de Crecimiento: $\mathrm{A}=$ árbol; $\mathrm{Ar}=$ arbusto; $\mathrm{h}=$ hierba; $\mathrm{th}=$ trepadora herbácea; $\mathrm{tl}=$ trepadora leñosa.

FAMILIA

Especie

Localidad

Tipo de

Forma de

potrero crecimiento

\section{PTERIDOPHYTA}

ADIANTACEAE

Hemionitis palmata $\mathrm{L}$.

Pityrogramma calomelanos (L.) Link

$\mathrm{B}, \mathrm{LP}$

G, E

$\mathrm{h}$

DAVALLIACEAE

Nephrolepis multiflora (Roxb.) F. M. Jarrett ex

$\mathrm{B}$

$\mathrm{G}, \mathrm{E}$

$\mathrm{h}$

C. V. Morton

B, LP $\quad$ G, E $\quad h$

SCHIZAEACEAE

Lygodium heterodoxum Kunze

Lygodium venustum $\mathrm{Sw}$.

Lygodium sp.

SELAGINELLACEAE

Selaginella hoffmannii Hieron.*

B, LP

G, E

$\mathrm{h}$

THELYPTERIDACEAE

Macrothelypteris torresiana (Gaudich.) Ching

B G, E h

Thelypteris dentata (Forsskal) E. P. St. John

$\mathrm{B}, \mathrm{LP}$

$\mathrm{G}, \mathrm{E}$

h

Thelypteris meniscioides (Liebm.) C. F. Reed

B, LP

$\mathrm{G}, \mathrm{E}$

th

Thelypteris (Amauropelta) sp. (1) $\dagger$

$\mathrm{B}, \mathrm{LP}$

$\mathrm{G}, \mathrm{E}$

th

Thelypteris (Goniopteris) sp. (2) $\dagger$

$\mathrm{B}$

$\mathrm{E}$

th

Thelypteris (Goniopteris) sp. (3) $\dagger$

B

G, E

$\mathrm{E}$

$\mathrm{h}$

Thelypteris (Goniopteris) sp. (4)†

B

$\mathrm{h}$

\section{ANGIOSPERMAE}

\section{MAGNOLIOPSIDA}

\section{ACANTHACEAE}

Blechum brownei (L.) Ant. Juss.

Blechum sp.

B, LP

B
G, E

G $\mathrm{h}$

h 
Apéndice. Continuación.

FAMILIA

Especie

Localidad

Tipo de

Forma de

potrero

crecimiento

\section{AMARANTHACEAE}

Achyranthes aspera L.

Achyranthes indica (L.) Mill.

$\begin{array}{ccc}\text { B } & \text { E } & \text { h } \\ \text { B, LP } & \text { G } & \text { h } \\ \text { B, LP } & \text { G, E } & \text { h }\end{array}$

Iresine diffusa Humb. \& Bonpl.

APIACEAE

Eryngium foetidum L.

B

Género no determinado

LP

G

$\mathrm{h}$

APOCYNACEAE

Prestonia guatemalensis Woodson

Stemmadenia donnell-smithii (Rose) Woodson

Tabernaemontana alba Mill.

$\begin{array}{ccc}\text { B } & \text { G } & \text { tl } \\ B & \text { G, E } & \text { A } \\ B & \text { G } & \text { A }\end{array}$

ARISTOLOCHIACEAE

Aristolochia ovalifolia Duch.

ASCLEPIADACEAE

Asclepias curassavica L.

B, LP

LP

Gonolobus sp.

Marsdenia macrophylla (Kunth) Fourn.

LP

LP

B, LP

$\mathrm{G}, \mathrm{E}$

$\mathrm{G}, \mathrm{E}$

$\mathrm{E}$

E

h

h

$\mathrm{h}$

ASTERACEAE

Acmella sp.

Ageratina pazcuarensis (Kunth) R. M. King \& H. Rob. *

Ageratum houstonianum Mill.

LP

Aster subulatus Michx.

B

Bidens odorata Cav.

LP

Calyptocarpus vialis Less.

B, LP

B, LP

B, LP

B

B, LP

E

G

A

Chaptalia nutans (L.) Pol.

Conyza apurensis Kunth *

Conyza canadensis (L.) Cronquist*

Critonia quadrangularis (DC.) R. M. King \&

H. Rob. *

Delilia berteri Spreng. *

$\mathrm{B}, \mathrm{LP}$

Eclipta alba Hassk. *

B

Epaltes mexicana Less. *

$\mathrm{B}$

Erechtites hieraciifolia (L.) Raf. ex DC.

$\mathrm{B}$

G, E

$\mathrm{G}, \mathrm{E}$

$\mathrm{G}, \mathrm{E}$

tl

$$
\begin{aligned}
& \mathrm{tl} \\
& \mathrm{A} \\
& \mathrm{A} \\
& \mathrm{tl}
\end{aligned}
$$

$$
\text { A }
$$

$\mathrm{h}$

$\mathrm{h}$

tl

G

G, E h


Apéndice. Continuación.

FAMILIA

Especie

Localidad

Tipo de

Forma de

potrero crecimiento

Eupatorium sp.

$\mathrm{B}$

E $h$

Hebeclinium macrophyllum (L.) DC. *

B

Heliopsis buphthalmoides Jacq. *

B, LP

E h

Melampodium divaricatum (Rich.) DC.

B, LP

G, E

$\mathrm{h}$

Mikania cordifolia (L. f.) Willd.

$\mathrm{B}, \mathrm{LP}$

E

h

Neurolaena macrocephala Sch. Bip. ex Hemsl.

$\mathrm{B}$

G, E

th

Neurolaena sp.

Porophyllum ruderale (Jacq.) Cass.

B $\quad$ E $h$

Pseudelephantopus spicatus (Aubl.) Rohr

B, LP

E

B, LP

G, E

LP

LP

G

Vernonia patens Kunth

B

E

Youngia japonica (L.) DC.

E

\section{(1)}

BIGNONIACEAE

Paragonia pyramidata (Rich.) Bur.

Stizophyllum riparium (Kunth) Sandwith

B

G

E

tl

B

B

G

Ar

Cordia spinescens L.

B

$$
\text { G }
$$

tl

BURSERACEAE

Bursera simaruba (L.) Sarg.

B

G, E

A

CAESALPINIACEAE

Chamaecrista nictitans (L.) Moench

LP

Cynometra retusa Britton \& Rose

Senna cobanensis (Britton) H. S. Irwin \& Barneby *

Senna multijuga var. doylei (Britton) H. S. Irwin \& Baneby

Senna papillosa (Britton \& Rose) H. S. Irwin \& Barneby

B

B, LP

E

$\mathrm{h}$

CARYOPHYLLACEAE

Arenaria lanuginosa (Michx.) Rohrb.

B, LP

G

A

Drymaria cordata (L.) Willd. ex Roem \&

LP

$\mathrm{B}, \mathrm{LP}$

G, E

$\mathrm{h}$ Schult.

Stellaria ovata Willd. \& Schltdl.

B, LP

G, E

h 
Apéndice. Continuación.

FAMILIA

Especie

Localidad

Tipo de

Forma de

potrero

crecimiento

\section{CECROPIACEAE}

Cecropia obtusifolia Bertol.

B

E

A

CONVOLVULACEAE

Ipomoea batatas (L.) Poir.

B, LP

G, E

th

Merremia umbellata (L.) Hallier f.

LP

G

th

Género no determinado

B, LP

G

CUCURBITACEAE

Melothria pendula L.

B, LP

G, E

$\mathrm{h}$

Momordica charantia L.

$\mathrm{B}$

E

th

EUPHORBIACEAE

Acalypha arvensis Poepp. \& Endl.

B, LP

G, E

$\mathrm{h}$

Acalypha diversifolia Jacq.

$\mathrm{B}$

$\mathrm{B}, \mathrm{LP}$

G, E

Ar

Adelia barbinervis Schltdl. \& Cham.

B

G, E

Ar

Caperonia castaneaefolia (L.) J. St.-Hil.

$\mathrm{E}$

Ar

Chamaesyce hirta (L.) Millsp.

B, LP

G, E

$\mathrm{h}$

Croton lobatus L.

B, LP

G, E

Dalechampia spathulata (Scheidw.) Baill.

$\mathrm{B}, \mathrm{LP}$

$\mathrm{G}, \mathrm{E}$

$\mathrm{h}$

Euphorbia heterophylla L.

$\mathrm{B}, \mathrm{LP}$

G, E

$\mathrm{h}$

LP

G

$\mathrm{h}$

Jatropha curcas L. *

B, LP

G, E

Ar

Phyllanthus amarus Schumach. \& Thonn. *

$\mathrm{B}, \mathrm{LP}$

$\mathrm{G}, \mathrm{E}$

$\mathrm{h}$

Phyllanthus compressus Kunth *

$\mathrm{B}, \mathrm{LP}$

G, E

h

Phyllanthus urinaria L. *

B

Género no determinado 1

$\mathrm{E}$

Ar

B, LP

G

A

Género no determinado 2

LP

Género no determinado 3

B, LP

G

$\mathrm{h}$

$\mathrm{h}$

FABACEAE

Aeschynomene americana L.

B, LP

G, E

G

h

Calopogonium mucunoides Desv.

$\mathrm{B}, \mathrm{LP}$

G, E

$\mathrm{h}$

Crotalaria bupleurifolia Cham. \& Schltdl. *

B

$\mathrm{G}$

th

Desmodium adscendens (Sw.) DC.

B, LP

G, E

$\mathrm{h}$

Desmodium affine Schltdl.

LP

E

h

Desmodium axillare (Sw.) DC.

$\mathrm{B}, \mathrm{LP}$

G, E

$\mathrm{h}$

Desmodium incanum DC.

$\mathrm{B}, \mathrm{LP}$

$\mathrm{G}, \mathrm{E}$

$\mathrm{h}$

h 
Apéndice. Continuación.

FAMILIA

Especie

Localidad

Tipo de

Forma de

potrero crecimiento

Desmodium macrodesmum (S. F. Blake) Standl.

B

G

th

\& Steyerm.

Desmodium scorpiurus (Sw.) Desv.

B, LP

G, E

$\mathrm{h}$

Desmodium tortuosum (Sw.) DC.

LP

E

B, LP

G, E

$\mathrm{h}$

Desmodium triflorum (L.) DC. *

LP

E

$\mathrm{h}$

Lonchocarpus cruentus Lundell

LP

B

G

A

Lunania mexicana Brandegee

LAMIACEAE

Hyptis atrorubens Poit.

$\mathrm{B}, \mathrm{LP}$

G, E

A

Hyptis capitata Jacq.

Hyptis mutabilis (A. Rich.) Briq.

B

B

Hyptis verticillata Jacq. *

LP

Salvia occidentalis Sw.

$\mathrm{B}, \mathrm{LP}$

E

G

E

G, E

G, E

$\mathrm{B}, \mathrm{LP}$

$\mathrm{G}, \mathrm{E}$

Género no determinado 1

Género no determinado 2

LP

G, E

$\mathrm{h}$

B

$\mathrm{G}, \mathrm{E}$

LOGANIACEAE

Spigelia palmeri Rose

B

E

$\mathrm{h}$

LYTHRACEAE

Cuphea carthagenensis (Jacq.) J. F. Macbr.

B

G

h

MALPIGHIACEAE

Stigmaphyllon lindenianum A. Juss.

B, LP

G, E

th

MALVACEAE

Herissantia crispa (L.) Brizicky

B, LP

G, E

B, LP

G, E

$\mathrm{h}$

Pavonia schiedeana Steud.

$\mathrm{B}, \mathrm{LP}$

$\mathrm{G}, \mathrm{E}$

B, LP

G, E

$\mathrm{h}$

h

Sida rhombifolia L.

Urena lobata L.

B

G

Ar

Clidemia petiolaris (Schltdl. \& Cham.) Schltdl. ex Triana

Conostegia xalapensis (Kunth) G. Don ex DC.

Tibouchina longifolia (Vahl) Baill. ex Cogn.

MIMOSACEAE

Acacia cornigera (L.) Willd.

B, LP

G, E

Ar 
Apéndice. Continuación.

FAMILIA

Especie

Localidad

Tipo de

Forma de

potrero

crecimiento

Mimosa floribunda Willd. *

$\mathrm{B}, \mathrm{LP}$

G, E

$\mathrm{h}$

Mimosa pudica L.

B, LP

G, E

$\mathrm{h}$

MONIMIACEAE

Siparuna andina (Tul.) A. DC.

B

E

Ar

MORACEAE

Trophis mexicana (Liebm.) Bureau

B

G

A

MYRTACEAE

Eugenia capuli (Schltdl. \& Cham.) O. Berg

B

G

A

Psidium guajava L. *

$\mathrm{B}, \mathrm{LP}$

G, E

A

ONAGRACEAE

Ludwigia octovalvis (Jacq.) P. H. Raven

B

E

$\mathrm{h}$

OXALIDACEAE

Oxalis corniculata L.

B, LP

G, E

$\mathrm{h}$

PASSIFLORACEAE

Passiflora coriacea A. Juss.

B

Passiflora sp.

B, LP

G

th

PHYTOLACCACEAE

Rivina humilis L.

B

E

th

PIPERACEAE

Piper aequale Vahl

Piper hispidum Sw.

Pothomorphe peltata (L.) Miq.

B

B, LP

B

Pothomorphe umbellata (L.) Miq.

B, LP

G

$\mathrm{h}$

RANUNCULACEAE

Clematis dioica $\mathrm{L}$.

B

G

Ar

RUBIACEAE

Borreria laevis (Lam.) Griseb.

$\mathrm{B}, \mathrm{LP}$

G, E

Ar

Crusea hispida (Mill.) B. L. Rob.

$\mathrm{B}, \mathrm{LP}$

Diodia maritima Thonn. *

LP

$\mathrm{G}, \mathrm{E}$

h

Diodia ocymifolia Bremek. *

B

B

G, E

$\mathrm{h}$

Geophila macropoda (Ruíz \& Pav.) DC.

$\mathrm{B}, \mathrm{LP}$

G

tl

Mitracarpus villosus (Sw.) Cham. \& Schltdl.

B, LP

G, E

$\mathrm{E}$

E

$\mathrm{B}, \mathrm{LP}$

$\mathrm{G}, \mathrm{E}$

G, E

$\mathrm{G}, \mathrm{E}$

G, E

$\mathrm{G}, \mathrm{E}$

G, E

h

$\mathrm{h}$

$\mathrm{h}$

$\mathrm{B}$

$\mathrm{h}$

$\mathrm{h}$

$\mathrm{h}$

$\mathrm{h}$

Spermacoce riparia Cham. \& Schltdl.

Género no determinado

$\mathrm{h}$

$\mathrm{h}$ 
Apéndice. Continuación.

FAMILIA

Especie

Localidad

Tipo de

Forma de

potrero crecimiento

RUTACEAE

Citrus aurantium L. *

B, LP

G, E

A

Citrus limon (L.) Burm. f. *

$\mathrm{B}, \mathrm{LP}$

G, E

A

Zanthoxylum kellermanii P. G. Wilson

B

$\mathrm{G}$

A

SAPINDACEAE

Cardiospermum sp. *

Cupania glabra Sw.

Paullinia clavigera Schltdl.

Paullinia venosa Radlk.

Serjania mexicana (L.) Willd.

$\begin{array}{lll}\text { B } & \text { G } & \text { h } \\ \text { B } & \text { E } & \text { A } \\ \text { B } & \text { G } & \text { tl } \\ \text { B } & \text { G } & \text { tl } \\ \text { B } & \text { E } & \text { tl }\end{array}$

SCROPHULARIACEAE

Bacopa procumbens Greenm. *

B, LP G, E h

Lindernia diffusa (L.) Wettst. *

$\mathrm{B}, \mathrm{LP}$

G, E

$\mathrm{h}$

SOLANACEAE

Cestrum sp.

B E

Physalis gracilis Miers

Physalis pubescens L.

B, LP

G, E

$\mathrm{B}$

G, E

B, LP

G, E

A

tl

tl

tl

Solanum americanum Mill.

B

E

$\mathrm{B}, \mathrm{LP}$

G, E

B, LP

$\mathrm{G}$

Ar

TILIACEAE

Trichospermum mexicanum (DC.) Baill.

B

G

A

URTICACEAE

Myriocarpa longipes Liebm.

B

G,E

$\mathrm{Ar}$

Urera caracasana (Jacq.) Griseb.

B

$\mathrm{G}$

Ar

VERBENACEAE

Lantata hirta Gram.

Priva lappulacea (L.) Pers.

B $G \quad h$

B G, E h

Stachytarpheta jamaicensis (L.) Vahl •

B

E

$\mathrm{h}$

Tamonea curassavica (L.) Pers. *

LP

E

$\mathrm{h}$

VIOLACEAE

Hybanthus attenuatus Schulze-Menz

B, LP

G, E

$\mathrm{h}$

VITACEAE

Cissus microcarpa Vahl

B, LP

G, E

tl/th 
Apéndice. Continuación.

FAMILIA

Especie

Localidad

Tipo de

Forma de

potrero

crecimiento

\section{LILIOPSIDA}

ARACEAE

Philodendron sp.

Syngonium podophyllum Schott

B $\mathrm{E} \quad \mathrm{h}$

Xanthosoma robustum Schott

B G, E h

COMMELINACEAE

Commelina diffusa Burm. f.

B, LP

G, E

G

$\mathrm{h}$

B

$\mathrm{h}$

Tripogandra floribunda (Hook. \& Arn.)

B, LP

G, E

$\mathrm{h}$

Woodson

Tripogandra serrulata (Vahl) Handlos

B, LP

G, E

$\mathrm{h}$

CYPERACEAE

Cyperus laxus Lam.

B, LP

G, E

$\mathrm{h}$

Cyperus tenuis Sw.

B, LP

G, E

$\mathrm{h}$

Cyperus sp.

$\mathrm{B}, \mathrm{LP}$

G, E

$\mathrm{h}$

Fimbristylis dichotoma (L.) Vahl

$\mathrm{B}, \mathrm{LP}$

$\mathrm{G}, \mathrm{E}$

$\mathrm{h}$

Kyllinga brevifolia Rottb.

$\mathrm{B}, \mathrm{LP}$

G, E

$\mathrm{h}$

Rhynchospora radicans (Schltdl. \& Cham.) H.

B, LP

G, E

$\mathrm{h}$

Pfeiff.

Scleria melaleuca Reichb. ex Cham. \& Schltdl.

DIOSCOREACEAE

Dioscorea composita Hemsl.

B, LP

$\mathrm{G}, \mathrm{E}$

$\mathrm{h}$

IRIDACEAE

Eleutherine latifolia (Stand1. \& L. O. Williams)

Ravenna

MARANTACEAE

Calathea lutea (Aubl.) G. Mey.

B

G, E

tl

ORCHIDACEAE

Erythrodes lunifera (Schltr.) Ames

B

B, LP

G, E

$\mathrm{h}$

Género no determinado

B

LP

E

$\mathrm{E}$

h

POACEAE

Acroceras zizanioides (Kunth) Dandy *

B, LP

G, E

h

Axonopus affinis Chase *

B, LP

G, E

h 
Apéndice. Continuación.

FAMILIA

Especie
Localidad

Tipo de

Forma de

potrero

\begin{tabular}{|c|c|c|c|}
\hline Axonopus compressus P. Beauv. * & $\mathrm{B}, \mathrm{LP}$ & $\mathrm{G}, \mathrm{E}$ & $\mathrm{h}$ \\
\hline Brachiaria distachya Stapf * & $\mathrm{B}, \mathrm{LP}$ & $\mathrm{G}, \mathrm{E}$ & $\mathrm{h}$ \\
\hline Brachiaria fasciculata (Sw.) Parodi & $\mathrm{B}$ & $\mathrm{E}$ & $\mathrm{h}$ \\
\hline Cynodon dactylon Pers. * & $\mathrm{B}, \mathrm{LP}$ & $\mathrm{G}, \mathrm{E}$ & $\mathrm{h}$ \\
\hline Cynodon plectostachyus Pilg. * & $\mathrm{B}, \mathrm{LP}$ & $\mathrm{G}, \mathrm{E}$ & h \\
\hline Digitaria bicornis (Lam.) Roem. \& Schult. * & $\mathrm{B}, \mathrm{LP}$ & $\mathrm{G}, \mathrm{E}$ & $\mathrm{h}$ \\
\hline Digitaria ciliaris (Retz.) Koeler * & $\mathrm{B}, \mathrm{LP}$ & $\mathrm{G}, \mathrm{E}$ & $\mathrm{h}$ \\
\hline Echinochloa colona (L.) Link & $\mathrm{B}, \mathrm{LP}$ & $\mathrm{G}$ & $\mathrm{h}$ \\
\hline Eleusine indica (L.) Gaertn. & $\mathrm{B}, \mathrm{LP}$ & $\mathrm{E}$ & h \\
\hline Homolepis aturensis (Kunth) Chase & LP & $\mathrm{G}, \mathrm{E}$ & $\mathrm{h}$ \\
\hline Lithachne pauciflora (Sw.) P. Beauv. ex Poir. & $\mathrm{B}$ & $\mathrm{G}$ & h \\
\hline Oplismenus hirtellus (L.) P. Beauv. * & $\mathrm{B}$ & $\mathrm{E}$ & $\mathrm{h}$ \\
\hline Panicum laxum Sw. & $\mathrm{B}, \mathrm{LP}$ & $\mathrm{G}, \mathrm{E}$ & $\mathrm{h}$ \\
\hline Panicum pilosum $\mathrm{Sw}$ * & $\mathrm{B}, \mathrm{LP}$ & $\mathrm{G}, \mathrm{E}$ & h \\
\hline Panicum trichanthum Nees * & $\mathrm{B}$ & $\mathrm{G}, \mathrm{E}$ & $\mathrm{h}$ \\
\hline Paspalum botterii Chase * & $\mathrm{B}, \mathrm{LP}$ & $\mathrm{G}, \mathrm{E}$ & $\mathrm{h}$ \\
\hline Paspalum conjugatum Bergius & $\mathrm{B}, \mathrm{LP}$ & $\mathrm{G}, \mathrm{E}$ & h \\
\hline Paspalum notatum Flüggé * & $\mathrm{B}, \mathrm{LP}$ & $\mathrm{G}, \mathrm{E}$ & h \\
\hline Paspalum plicatulum Michx. * & $\mathrm{B}, \mathrm{LP}$ & $\mathrm{G}$ & $\mathrm{h}$ \\
\hline Paspalum setaceum Michx. * & LP & $\mathrm{G}$ & $\mathrm{h}$ \\
\hline Rottboellia exaltata L. f. * & LP & $\mathrm{E}$ & h \\
\hline Setaria geniculata Beauv. * & $\mathrm{B}, \mathrm{LP}$ & $\mathrm{G}, \mathrm{E}$ & $\mathrm{h}$ \\
\hline
\end{tabular}

